\title{
Development of Drug Loaded Nanoparticles Binding to Hydroxyapatite Based on a Bisphosphonate Modified Nonionic Surfactant
}

\author{
Jiabin Zhang, ${ }^{1}$ Xinrong Liu, ${ }^{1}$ Tongming Deng, ${ }^{1}$ Peng Yao, ${ }^{1}$ Heliang Song, \\ Shaobing Zhou, ${ }^{2}$ and Weili Yan ${ }^{1}$ \\ ${ }^{1}$ School of Life Sciences and Engineering, Southwest Jiaotong University, Chengdu 610031, China \\ ${ }^{2}$ School of Material Sciences and Engineering, Southwest Jiaotong University, Chengdu 610031, China
}

Correspondence should be addressed to Xinrong Liu; lxr@mail.swjtu.edu.cn and Weili Yan; yanweili@gmail.com

Received 12 January 2015; Accepted 16 March 2015

Academic Editor: Xuping Sun

Copyright @ 2015 Jiabin Zhang et al. This is an open access article distributed under the Creative Commons Attribution License, which permits unrestricted use, distribution, and reproduction in any medium, provided the original work is properly cited.

\begin{abstract}
This study aimed at development of drug loaded nanoparticles which could bind to hydroxyapatite (HA) to construct drug or growth factor releasing bone graft substitutes. To this end, the terminal hydroxyl group of a nonionic surfactant Brij 78 (polyoxyethylene (20) stearyl ether) was first modified with pamidronate (Pa). Using Pa-Brij 78 as both a surfactant and an affinity ligand to HA, three different $\mathrm{Pa}$ surface functionalized nanoparticles were prepared, named as solid lipid nanoparticles (Pa-SNPs), nanoemulsions (PaNEMs), and PLGA nanoparticles (Pa-PNPs). A model drug curcumin was successfully encapsulated in the three nanoparticles. The sizes of $\mathrm{Pa}-\mathrm{NEM}$ and $\mathrm{Pa}$-PNP were around $150 \mathrm{~nm}$ and the size of $\mathrm{Pa}-\mathrm{SNP}$ was around $90 \mathrm{~nm}$ with polydispersity indexes (PDIs) less than 0.20 . Drug encapsulation efficiencies of the three nanoparticles were all greater than $85 \%$. Furthermore, the order of binding affinity of the nanoparticles to HA was Pa-PNP > Pa-NEM = Pa-SNP. After lyophilization, the sizes of the three nanoparticles were increased about 0.5-2.0-fold but their binding affinities to HA were almost the same as the fresh prepared nanoparticles. In conclusion, a Pa-modified Brij 78 was synthesized and used for fabrication of a series of drug loaded nanoparticles to construct drug-eluting HA-based bone graft substitutes.
\end{abstract}

\section{Introduction}

Hydroxyapatite (HA), a mineral form of calcium apatite with the formula $\mathrm{Ca}_{5}\left(\mathrm{PO}_{4}\right)_{3}(\mathrm{OH})$, is the major inorganic component of bone and teeth in mammals [1]. Because the crystalline phase of natural bone is similar to HA, HA ceramics have been widely used as a substitute in bone grafts. The porosity of HA in the range of $100-200 \mu \mathrm{m}$ in diameter enables mesenchymal cell migration, adhesion, proliferation, and differentiation into osteoblasts and the structure is also the basis of osteoconductive characteristics of HA. The unique physical property of HA allows the growth of neovasculature and infiltration of osteogenic precursor cells during the bone growth process. However, a graft material that is only osteoconductive cannot transfer inductive simulative signals but rather acts only as a scaffold that supports the bone growth [2], so ideally exogenous bioactive small molecules or growth factors should be incorporated into the graft substitute. Furthermore, musculoskeletal diseases and disorders often require drug treatment at the specific sites of surgery or defect [3]. In bone tissue engineering and treatment, the drugs could be not only traditional drugs such as antibiotic, anticancer, anti-inflammatory, but also biological agents, such as proteins and nucleic acids, which produce exogenous osteoinductive signals [4]. Thus, it is an urgent need to design drug and/or growth factor releasing HA for the bone grafts and repair.

There are several approaches to incorporate drugs or growth factors into HA. For example, the growth factors can be chemically modified to have the binding affinity to HA [5] and the small molecule drugs can be physically absorbed onto the surface of calcium phosphate based biomaterials [6]. These approaches rely on the fixed and limited surface of HA. Moreover, the incorporation of drugs into HA requires the specific chemical or physical properties of the drugs, so it 
is impossible to utilize a universal method to fabricate drug loaded and releasing HA. Furthermore, the highly hydrophobic drugs are difficult to be incorporated into HA crystal.

Drugs or growth factors releasing from bone graft substitutes should be in a controlled and sustained manner [7-11], and this can be achieved by nanoparticles (NPs) drug delivery system. Thus, the combination of drug loaded NPs with HA is a promising approach to prepare drug-eluting HA and keep both advantages of NPs and HA. First, the binding affinity between NPs and HA is majorly determined by their surface properties but is independent of the chemical or physical properties of drugs inside of NP, so theoretically the complex (NP-HA) formed by NPs and HA can incorporate drugs with a broad range of physical and chemical characteristics. Second, the NP system could virtually overcome the limitation of the HA surface to increase the drug loading efficiency of HA. Third, the matrix of NPs provides a diffusion barrier for the release of drugs and growth factors and the researchers are able to adjust the rate of the release by rational design of the components of the matrix of the NPs.

The structure of bisphosphonates (BP) presents two phosphonate groups sharing a common carbon atom (P-C$\mathrm{P})$, which are believed to be responsible for its high affinity to bone and HA. BP was utilized for the modification of the surfaces of micelles, liposomes, and NPs in the literature for bone targeting [12-15]. For example, the BP modified cholesterol, DSPE-PEG2000, and BPA have been used to prepare liposomes and micelles targeting bone [16]. Furthermore, de Miguel et al. synthesized PBLG-b-PEG-BP to prepare bone targeted NPs and achieved good results [13]. Thamake et al. prepared PLGA NPs and then coated BP on the surface of NPs resulting in satisfying bone targeting effects [15]. Theoretically, all those BP decorated bone targeted NPs could also be used for fabrication of drug releasing HA bone grafts substitutes. However, it is favorable to design a universal surfactant compound for fabrication of multiple types of NPs to satisfy the requirements of bone tissue engineering and treatment.

To this end, we synthesized a BP (e.g., pamidronate, Figure 1(a)) modified nonionic surfactant Brij 78 (Pa-Brij 78). Using $\mathrm{Pa}$-Brij 78, three different $\mathrm{Pa}$ surface functionalized NPs (Pa-NPs) were then prepared successfully including E-wax based solid lipid NPs (Pa-SNPs), glycerol trioctanoate based nanoemulsions (Pa-NEMs), and PLGA based biodegradable NPs (Pa-PNPs) [11-15]. We then studied the interactions between the Pa-NPs and HA and the properties of the NP-HA complex.

\section{Material and Methods}

2.1. Materials. Brij 78, 4-toluene sulfonyl chloride, triethylamine, pamidronate disodium, sodium bicarbonate, TPGS, glycerol trioctanoate, PLGA, and curcumin were purchased from Sigma (St. Louis, MO). E-wax was provided by Spectrum (New Brunswick, NJ). Hydroxyapatite was provided by Sinopharm Group (Shanghai, China).

2.2. Synthesis of Ts-Brij 78. Brij $78(1.15 \mathrm{~g}, 1 \mathrm{mmol})$ was first dissolved in $15 \mathrm{~mL}$ dichloromethane (DCM) and incubated at $0^{\circ} \mathrm{C}$ and 4-methyl-benzenesulfonyl chloride $(380 \mathrm{mg}$,
$2 \mathrm{mmol}$ ) and triethylamine $(303 \mathrm{mg}, 3 \mathrm{mmol}$ ) premixed in $5 \mathrm{~mL}$ DCM were added to the Brij 78 solution dropwise. The mixture was stirred for $18 \mathrm{~h}$ at room temperature, followed by rotary evaporation under reduced pressure, and the precipitate was redissolved in $65 \mathrm{~mL}$ ethanol acidified with $250 \mu \mathrm{L} 1 \mathrm{M} \mathrm{HCl}$. The solution was kept overnight at $-20^{\circ} \mathrm{C}$ and then the formed precipitate was centrifuged at $5000 \mathrm{~g}$ at $-10^{\circ} \mathrm{C}$. The solid Ts-Brij 78 was stored at $-20^{\circ} \mathrm{C}$.

2.3. Synthesis of $\mathrm{Pa}$-Brij 78. Ts-Brij 78 (1.3 g, $1 \mathrm{mmol})$, pamidronate disodium ( $370 \mathrm{mg}, 1 \mathrm{mmol}$ ), and sodium bicarbonate (420 mg, $5 \mathrm{mmol}$ ) were dissolved in $20 \mathrm{~mL}$ water. The mixture was refluxed for $24 \mathrm{~h}$, after which the solution was evaporated under reduced pressure and the precipitate was redissolved in $30 \mathrm{~mL}$ ethanol. The solution was filtered, the residue was discarded, and the filtrate was rotary evaporated under reduced pressure; then the precipitate was washed by petroleum ether three times and the solid was dried under vacuum. The solid Pa-Brij 78 was stored at room temperature.

2.4. Determination of Critical Micelle Concentration (CMC) of $\mathrm{Pa}$-Brij 78. The electrical conductivity method was widely used to estimate the degree of ionization $(\alpha)$ of ionic micelles and was used in determining the CMC of ionic surfactant [17]. To determine the CMC of Pa-Brij 78, different concentrations of Pa-Brij 78 aqueous solutions (0.1-3.9 mM) were prepared and their molar conductivities were measured at $25^{\circ} \mathrm{C}$ by a software of a particle size analyzer (Zetasizer NanoZS, Malvern Instruments Ltd, Malvern, UK). The graph of molar conductivities versus concentrations was plotted on which the turning point is the CMC of Pa-Brij 78.

\subsection{Preparation of Curcumin Loaded NPs Using Pa-Brij 78}

2.5.1. Curcumin Loaded Solid Lipid NPs. The method of preparation of E-wax based solid lipid NPs was based on previous reports $[18,19]$ with slight modification. Briefly, $4 \mathrm{mg}$ of E-wax, $7 \mathrm{mg}$ of Pa-Brij 78, or the mixture of Brij 78 and Pa-Brij 78 and $0.4 \mathrm{mg}$ of a model drug curcumin were mixed in a glass vial and then heated at $65^{\circ} \mathrm{C}$ and then $200 \mu \mathrm{L} 65^{\circ} \mathrm{C}$ distilled water was added to the vial. The mixture was stirred for $10 \mathrm{~min}$ at $65^{\circ} \mathrm{C}$ to form a slurry-like mixture. Then, $1.8 \mathrm{~mL}$ distilled water at $65^{\circ} \mathrm{C}$ was added to the vial heated at $65^{\circ} \mathrm{C}$; the mixture was continued stirring at $65^{\circ} \mathrm{C}$ for $40 \mathrm{~min}$ to form a warm clear oil-in-water microemulsion. NPs were obtained by cooling the vials to room temperature while stirring. The size and PDI of the NPs were measured by a particle size analyzer (Zeta sizer Nano-ZS, Malvern Instruments Ltd, Malvern, UK).

2.5.2. Curcumin Loaded Nanoemulsion. The method of preparation was adapted from previous work [18] with slight modification. Briefly, $2 \mathrm{mg}$ of glycerol trioctanoate as the oil phase, $3.5 \mathrm{mg}$ of $\mathrm{Pa}-\mathrm{Brij}$ 78, or the mixture of Brij 78 and $\mathrm{Pa}-$ Brij 78 in some experiments as surfactants, $1.2 \mathrm{mg}$ of TPGS as a cosurfactant, and $0.6 \mathrm{mg}$ of curcumin as a model drug were added and then heated to $55^{\circ} \mathrm{C}$ followed by addition of distilled water $\left(200 \mu \mathrm{L}, 55^{\circ} \mathrm{C}\right)$ at $55^{\circ} \mathrm{C}$. The mixture was stirred thoroughly for $10 \mathrm{~min}$ at $55^{\circ} \mathrm{C}$ to form a slurry-like mixture. 


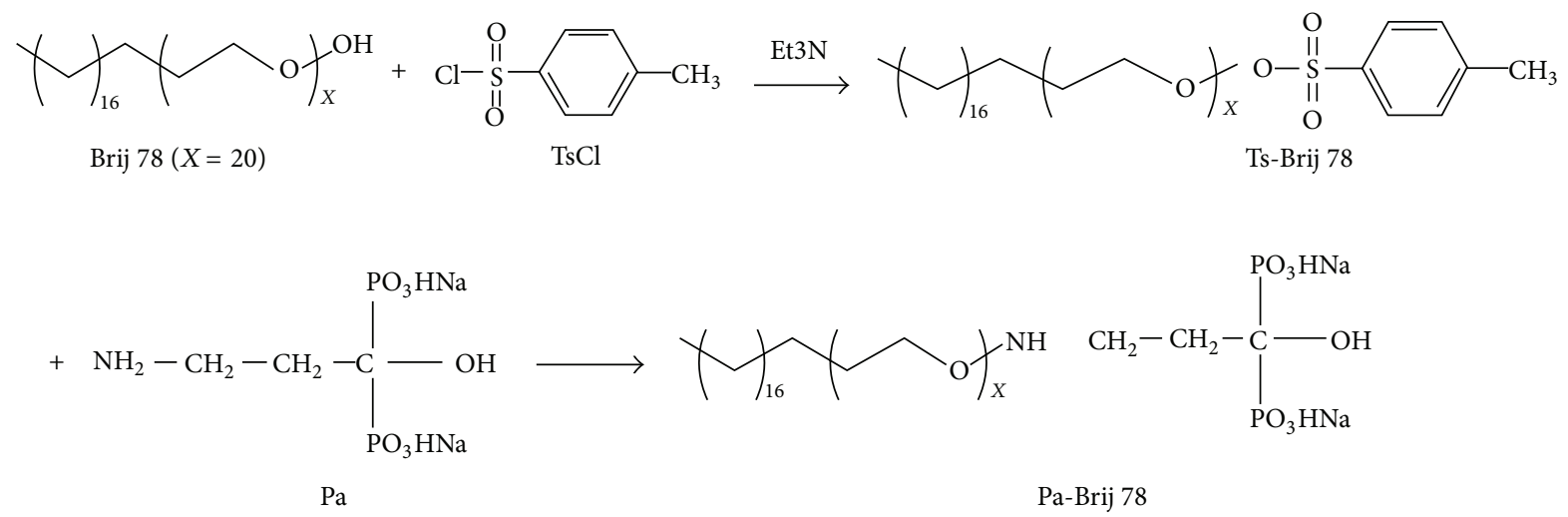

(a)

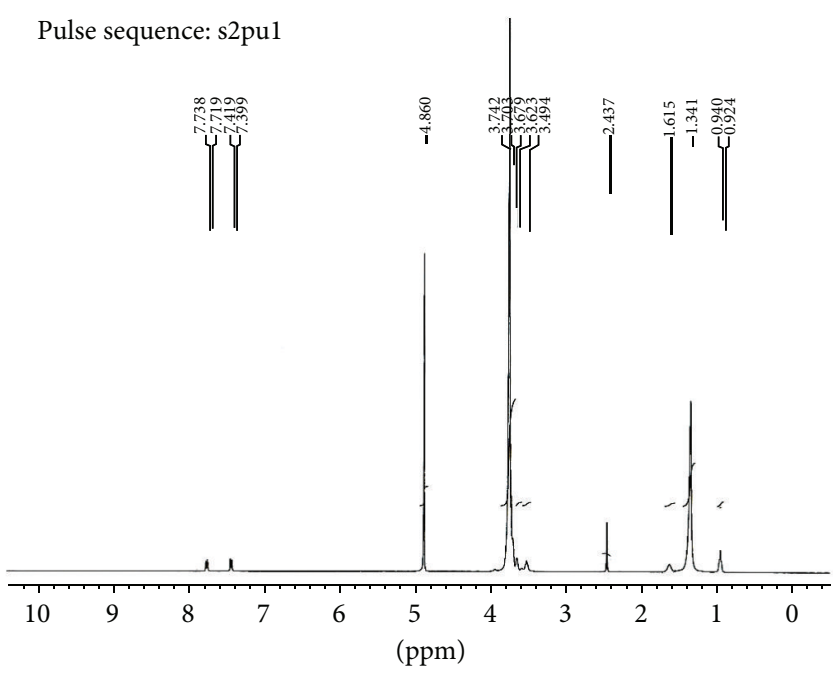

(b)

FIgURE 1: Synthesis and characterization of Pa-Brij 78. (a) Synthesis route scheme of Pa-Brij 78; (b) ${ }^{1} \mathrm{H}-\mathrm{NMR}$ of Pa-Brij 78.

Then, $1.8 \mathrm{~mL} 55^{\circ} \mathrm{C}$ distilled water was added to the vial at $55^{\circ} \mathrm{C}$ and the mixture was stirred at $55^{\circ} \mathrm{C}$ for at least $40 \mathrm{~min}$ to form a warm clear oil-in-water microemulsions. Nanoemulsions were obtained by cooling the vials to room temperature while stirring. The size and PDI of the NPs were measured by a particle size analyzer.

2.5.3. Curcumin Loaded PLGA NPs. The method of preparation was adapted from previous work $[16,17]$ with slight modification. Briefly, PLGA (20 mg) and $2 \mathrm{mg}$ of curcumin were dissolved in $1 \mathrm{~mL}$ DCM, and then the organic solution was poured into $4 \mathrm{~mL} 0.3 \% \mathrm{~Pa}-\mathrm{Brij} 78$ aqueous solution. The mixture was stirred vigorously for $5 \mathrm{~min}$ and then poured into the high pressure homogenizer (Model number JN-02C by Junbio, Guangzhou, China) with the pressure of 1500 bars at $4^{\circ} \mathrm{C}$ for 5 consecutive cycles to form a microemulsion. The DCM was removed from the microemulsion under reduced pressure to form the curcumin loaded PLGA NPs. The size and PDI of the NPs were measured by a particle size analyzer.

2.6. Curcumin Encapsulation in NPs. The method of determination of the entrapment efficiency of curcumin in the
NPs was adapted from literature [10] with slight modification. Briefly, ultrafiltration centrifuge tubes $(30 \mathrm{kD}$ MWCO from VWR) were used to separate free curcumin from the NPs. The concentration of curcumin in the filtrate after centrifugation was measured by a fluorescence method (excitation wavelength, $420 \mathrm{~nm}$; emission wavelength, $470 \mathrm{~nm}$ ). A standard curve was plotted using a set of standard concentrations of curcumin dissolved in ethanol. The amount of encapsulated curcumin and the encapsulation efficiency were calculated by the following equation:

$$
\begin{aligned}
\text { Curcumin Encapsulated }= & \text { Total curcumin input } \\
& - \text { the curcumin in the filtrate, } \\
\text { Encapsulation Efficiency }= & \left(\frac{\text { Curcumin Encapsulated }}{\text { Total Curcumin input }}\right) \\
& \times 100 \% .
\end{aligned}
$$

2.7. Scanning Electron Microscopy (SEM). Prior to the examination, the samples were deposited on a brass stub using a 
double-sided tape and stored at $-20^{\circ} \mathrm{C}$ and then the samples were freeze-dried for $12 \mathrm{~h}$. The samples were gold-coated for $200 \mathrm{~s}$ by a sputter coater. The morphologies of nanoparticles were examined using a Scanning Electron Microscope (JEOL JSM-7001F, Japan) operated at an accelerating voltage of $15 \mathrm{kV}$.

2.8. Binding Ratio of NPs or Pa-NPs to HA. First, the concentration of each NP was diluted to $1.0 \mathrm{mg} / \mathrm{mL}$ of matrix used. One $\mathrm{mL}$ of each of the curcumin loaded NPs (SNP, PaSNP, NEM, Pa-NEM, PNP, and Pa-PNP) and 5-200 mg dry powder of $\mathrm{HA}$ were mixed in $1.5 \mathrm{~mL}$ centrifuge tubes. The NPs and HA were incubated with gentle shaking for $5 \mathrm{~min}$ on the oscillator and then briefly centrifuged to separate unbound NPs from the complex of HA/Pa-NPs. The NPs in the supernatant were quantified by measurement of curcumin concentration by a fluorescence method mentioned in Section 2.6. For the binding kinetics study, $1 \mathrm{~mL}$ of each of the NPs described previously and $60 \mathrm{mg}$ HA were added to $1.5 \mathrm{~mL}$ centrifuge tubes and then incubated with gentle shaking for different time on the oscillator and centrifuged for separation of unbound NPs from HA; the unbound NPs in the supernatant were quantified by measurement of curcumin concentration by a fluorescence method mentioned in Section 2.6.

The NPs were also labeled by coumarin (10\% weight of curcumin) for fluorescence images. The phase contrast and fluorescence images were captured by an Olympus IX71 fluorescence microscope.

2.9. Specific Binding of $\mathrm{Pa}-\mathrm{NPs}$ to $\mathrm{HA}$. To study specificity of $\mathrm{Pa}-\mathrm{NPs}$ binding to $\mathrm{HA}$, the $\mathrm{HA} / \mathrm{Pa}-\mathrm{NPs}$ complex was constructed with the method mentioned in Section 2.7. The pamidronate disodium aqueous solution (10 $\mathrm{mM}$ or $50 \mathrm{mM})$ was then added to the complex of $\mathrm{HA} / \mathrm{Pa}-\mathrm{NP}$ followed by gentle shaking at $37^{\circ} \mathrm{C}$ for $2 \mathrm{~h}$ for competition. After centrifugation, the unbound NPs in the supernatant were quantified by measurement of curcumin concentration by a fluorescence method mentioned in Section 2.6.

2.10. Binding of $\mathrm{Pa}-\mathrm{NPs}$ to $\mathrm{HA}$ with the Existence of Free Calcium Chloride. One $\mathrm{mL}$ of each Pa-NPs and $60 \mathrm{mg} \mathrm{HA}$ were added to a centrifuge tube with or without the existence of 2.5 or $5 \mathrm{mM}$ calcium chloride. The centrifuge tube was incubated at $37^{\circ} \mathrm{C}$ with gentle shaking for $5 \mathrm{~min}$ on the oscillator and then centrifuged for separation of unbound NPs from the complex of HA/Pa-NPs. The amount of unbound NPs in the supernatant was quantified by measurement of curcumin concentration by a fluorescence method mentioned in Section 2.6.

2.11. Stability of the Complex of $\mathrm{HA} / \mathrm{Pa}-\mathrm{NPs}$ in Calcium Chloride Solution. The HA/Pa-NPs complex was prepared by the method mentioned in Section 2.7. The calcium chloride solution (final concentration equal to $2.5 \mathrm{mM}$ or $5.0 \mathrm{mM}$ ) was then added to the complex of HA/Pa-NP followed by gentle shaking at $37^{\circ} \mathrm{C}$ for $2 \mathrm{~h}$. After centrifugation, the amount of NPs in the supernatant was quantified by measurement of
TABLE 1: The particle size, PDI, and the surface charge of the nanoparticles.

\begin{tabular}{lccc}
\hline NPs & Mean size $(\mathrm{nm})$ & PDI & Zeta $(\mathrm{mV})$ \\
\hline Pa-SNPs & 83.9 & 0.144 & -13.0 \\
Pa-NEMs & 168.4 & 0.114 & -12.6 \\
Pa-PNPs & 156.7 & 0.105 & -15.6 \\
\hline
\end{tabular}

curcumin concentration by fluorescence method mentioned in Section 2.6.

2.12. The Freeze-Drying of NPs. Pa-NPs suspension was mixed with equal volume of lyoprotecting solution $(20 \%$ sucrose solution). Then the Pa-NPs were freeze-dried by a standard protocol. The freeze-dried samples were stored in $-80^{\circ} \mathrm{C}$ for a month and reconstructed in PBS buffer to obtain recovered $\mathrm{Pa}-\mathrm{NPs}$ suspensions. The recovered $\mathrm{Pa}-\mathrm{NPs}$ were first analyzed by size distribution and PDI and then were tested for binding affinity to HA and the results were compared with fresh prepared Pa-NPs.

\section{Results and Discussion}

3.1. Synthesis and Characterization of Pa-Brij 78. Brij 78 contains a hydrophilic PEG chain, so the hydroxyl end group of PEG was first activated by $\mathrm{TsCl}$ to form Ts-Brij 78. The $\mathrm{Pa}-\mathrm{Brij} 78$ was then successfully conjugated by reaction of amine group of $\mathrm{Pa}$ to the Ts-Brij 78, with the existence of sodium bicarbonate to keep the reaction under basic condition (Figure 1(a)). The overall yield achieved about $80 \%$. The structure of Pa-Brij 78 was confirmed by ${ }^{1} \mathrm{H}-\mathrm{NMR}$ (the solvent was $\left.\mathrm{D}_{2} \mathrm{O}\right)$. The signals $(7.73-7.39, \mathrm{~m}, 1 \mathrm{H}, 2.43, \mathrm{~s}, 2 \mathrm{H}$, and $1.61, \mathrm{~s}, 2 \mathrm{H}$ ) indicated the existence of a $-\mathrm{NH}-\mathrm{CH}_{2}-\mathrm{CH}_{2}-$ in this compound (Figure 1(b)). The CMC of Pa-Brij 78 was around $1.9-2.1 \mathrm{mM}$ at $25^{\circ} \mathrm{C}$ measured by the method of molar conductivity.

3.2. Preparation and Characterization of PA Functionalized NPs. Three types of $\mathrm{Pa}$ functionalized NPs (Pa-NPs) were successfully prepared. The size and surface charge of the NPs were analyzed by light scattering. The average particle sizes of the NPs were $83.9 \mathrm{~nm}$ for Pa-SNPs (Figure 2(a)), $168.4 \mathrm{~nm}$ for Pa-NEMs (Figure 2(b)), and $156.7 \mathrm{~nm}$ for Pa-PNPs (Figure 2(c)). The data of zeta potential indicated the negative charge on the surface of Pa-NPs. The SEM images clearly showed the sphere shape of Pa-NPs (Figures 3(a), 3(b), and 3(c)). The sizes, PDI, and zeta potential of the Pa-NPs were summarized in Table 1.

After storage for 7 days at $4^{\circ} \mathrm{C}$, the colloidal suspensions of $\mathrm{Pa}$-NPs kept the same appearance without precipitation or cloudiness. The PDIs and particle sizes of the Pa-NPs were monitored for 7 days and the data indicated only a small increase of the sizes $(<20 \%)$ during the 7-day storage (Figure 4). Furthermore, the PDIs of the Pa-NPs were all less than 0.25 during the 7-day storage suggesting the monodispersity of the particles. The good stability of the Pa-NPs probably was due to hydrophilic PEG shielding and a negative surface 


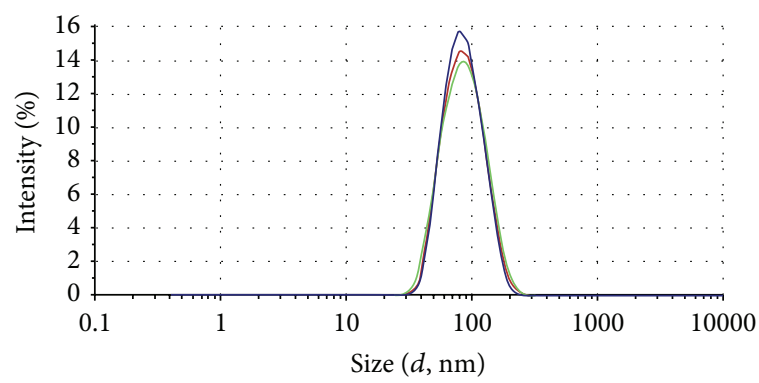

(a)

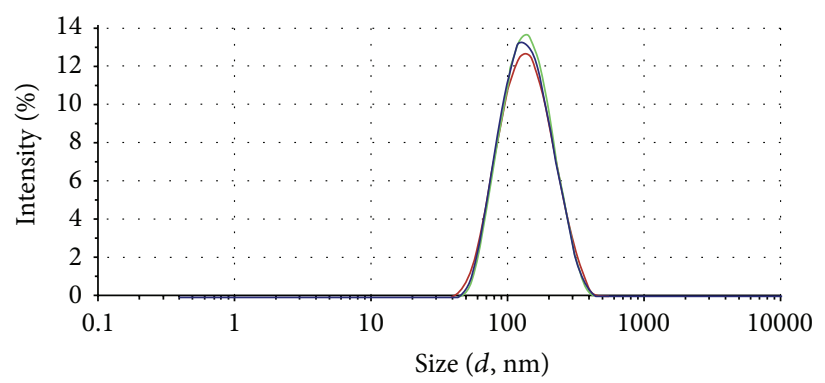

(b)

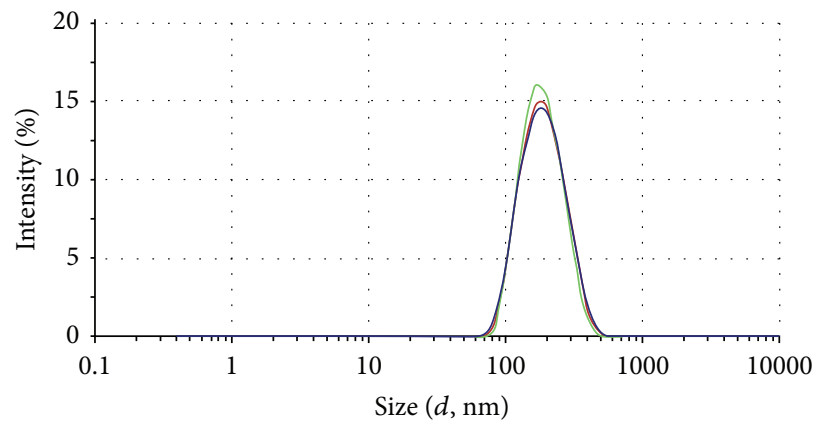

(c)

Figure 2: Particle size distribution of Pa-NPs by dynamic light scattering (DLS). (a) Pa-SNPs; (b) Pa-NEMs; (c) Pa-PNPs.

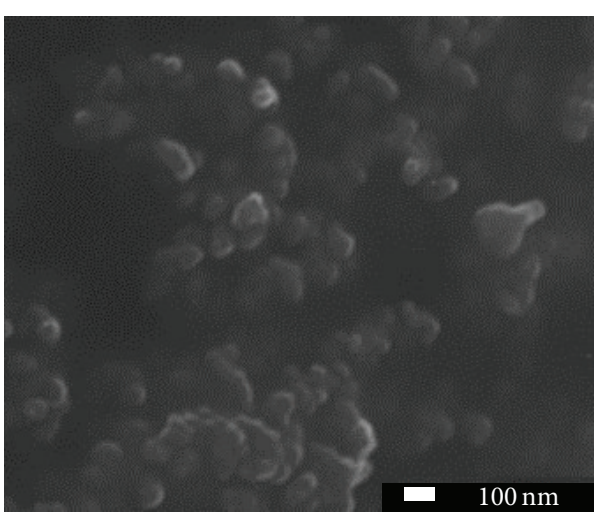

(a)

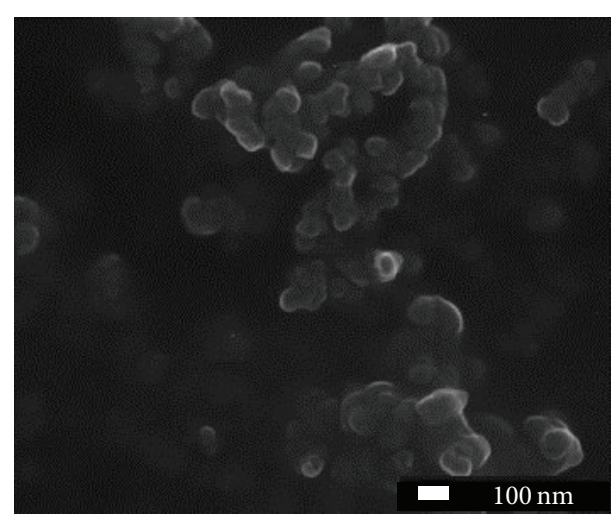

(b)

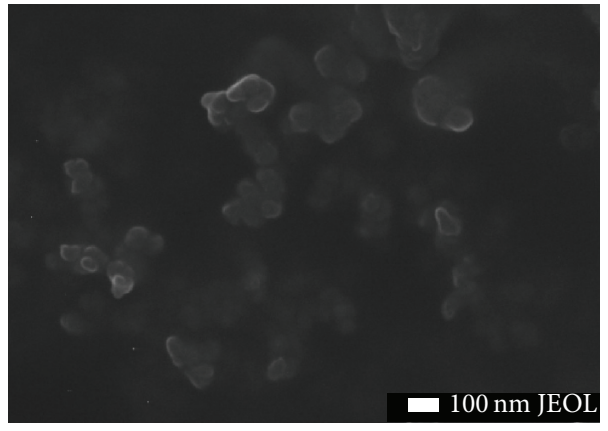

(c)

FIgure 3: The morphology of Pa-NPs characterized by SEM images. (a) Pa-SNPs; (b) Pa-NEMs; (c) Pa-PNPs. 


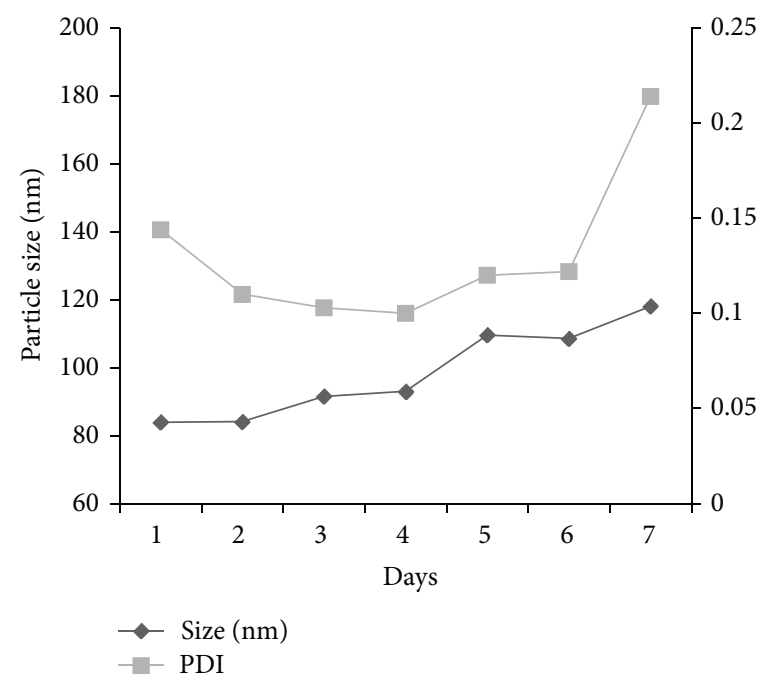

(a)

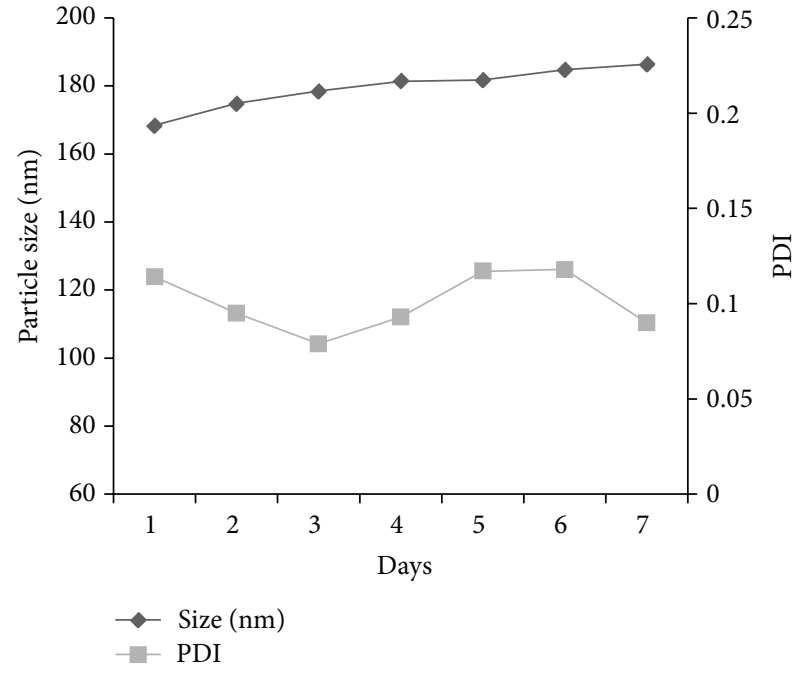

(b)

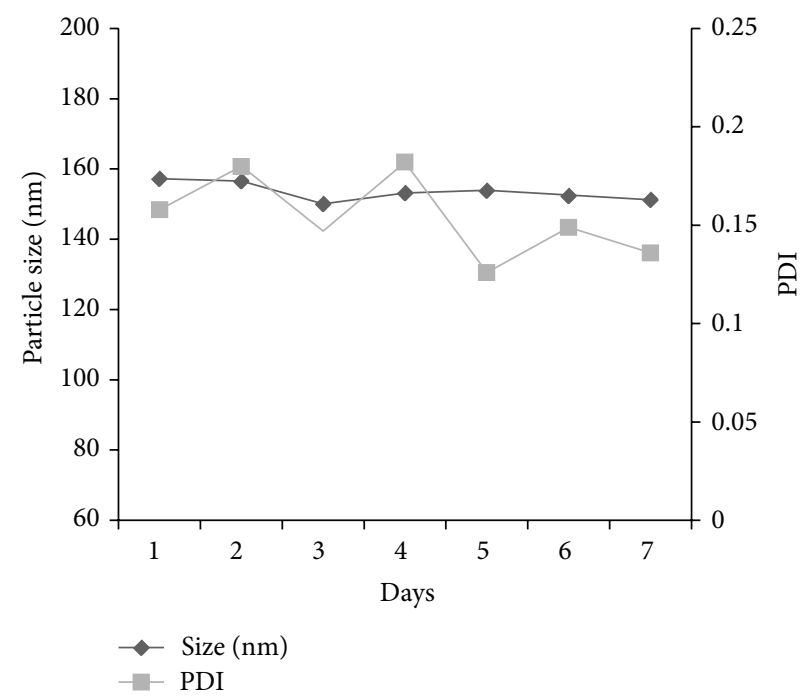

(c)

FIgure 4: The short-term stability of the three nanoparticles at $4^{\circ} \mathrm{C}$ for 7 days. (a) Pa-SNPs; (b) Pa-NEMs; (c) Pa-PNPs.

charge. The lipid based SNPs and NEMs were widely used for delivery of hydrophobic drugs to increase their solubility and bioavailability and the biodegradable PLGA based PNPs have the potential for delivery of hydrophilic drugs and protein or peptide based growth factors. Thus, Pa-Brij 78 has the capability to fabricate a series of Pa-NPs which can encapsulate therapeutic agents with a broad range of chemical or physical properties.

3.3. HA Binds to Curcumin Loaded Pa-NPs to Form Drug Loaded $\mathrm{HA} / \mathrm{Pa}-\mathrm{NPs}$ Complex. After the conditions of fabrication of Pa-NPs were optimized, a model drug curcumin was encapsulated in Pa-NPs. Table 2 showed that all the Pa-NPs could achieve more than $85.0 \%$ drug loading efficiency and that the drug encapsulation efficiency of the Pa-NPs was in the range of $10-30 \%$. The drug to carrier ratio of $\mathrm{Pa}-\mathrm{NEM}$ was greater than $30 \%$ because the hydrophobic drug curcumin
TABle 2: The drug encapsulation efficiency and drug loading efficiency of the nanoparticles.

\begin{tabular}{lcc}
\hline Nanoparticles & $\begin{array}{c}\text { Drug }^{*} \\
\text { encapsulation } \\
\text { efficiency (\%) }\end{array}$ & $\begin{array}{c}\text { Drug* loading } \\
\text { efficiency (\%) }\end{array}$ \\
\hline Pa-SNPs & 88.4 & 11.3 \\
Pa-NEMs & 85.6 & 32.5 \\
Pa-PNPs & 89.4 & 10.5 \\
\hline
\end{tabular}

${ }^{*}$ The curcumin was used as model drug.

might dissolve well in the liquid oil phase of emulsion to enhance the drug loading efficiency.

Then we continued to explore the conditions to build the drug loaded HA/Pa-NPs complex. First, the fixed amount 


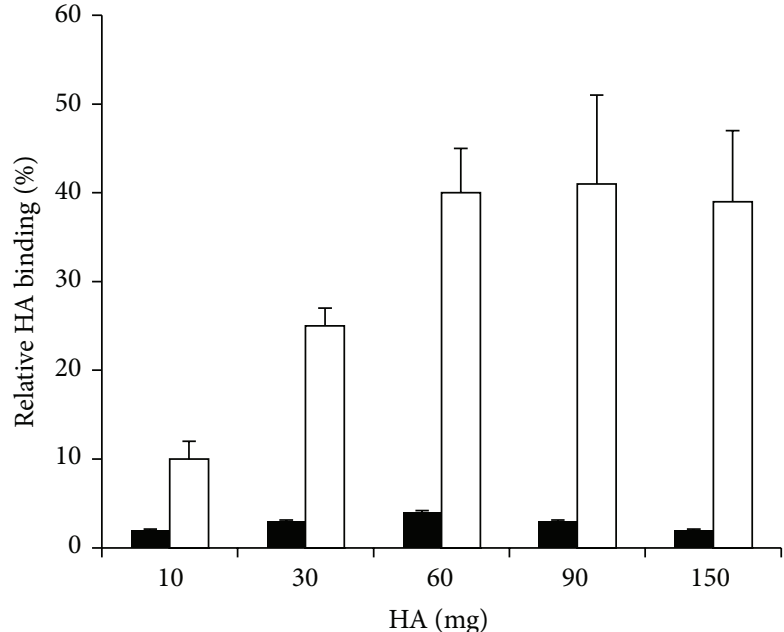

SNPs $\square$ Pa-SNPs

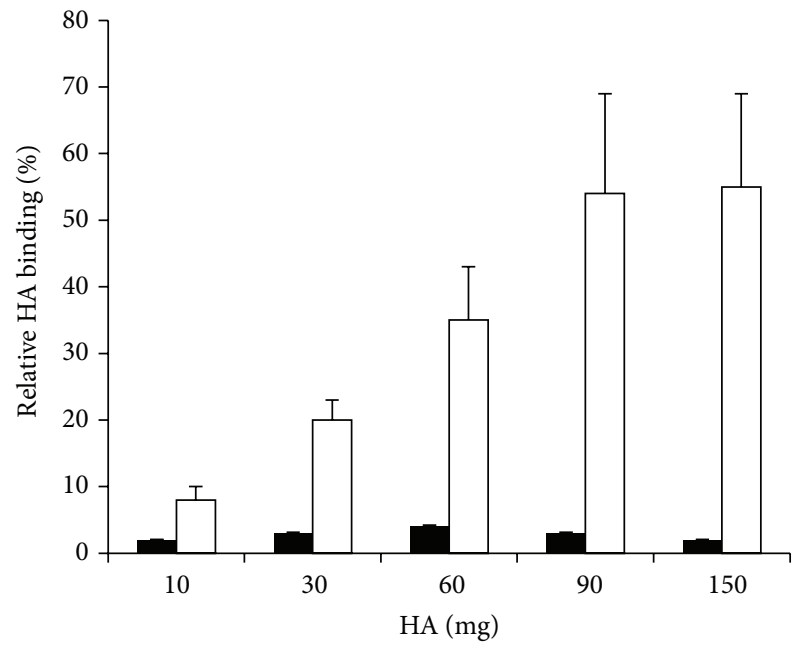

NEMs

Pa-NEMs

(a)

(b)

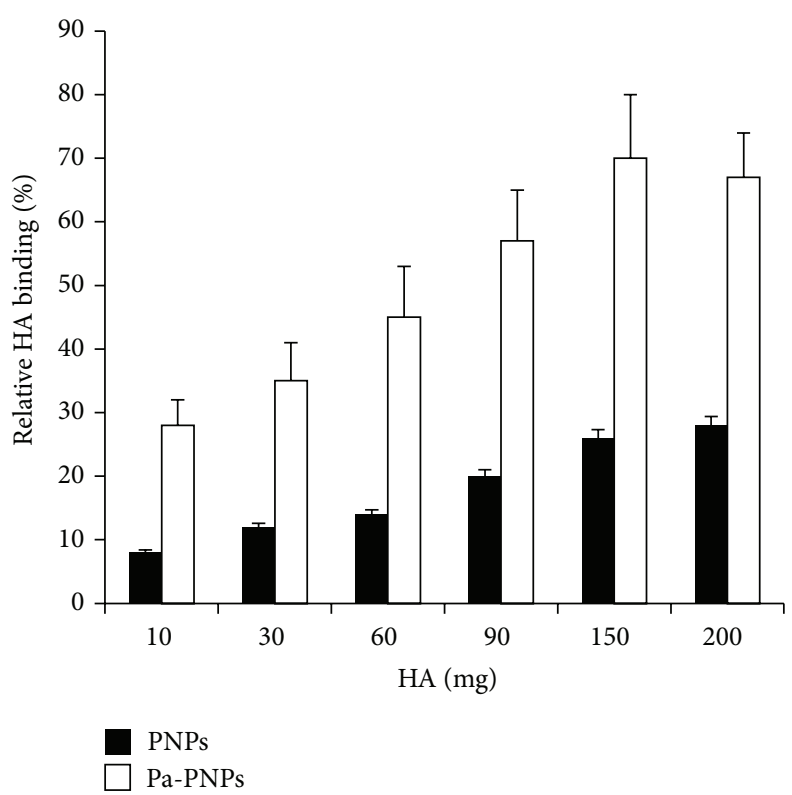

(c)

Figure 5: HA binding to Pa-NPs with different weight ratio of HA to NPs. (a) SNPs and Pa-SNPs; (b) NEMs and Pa-NEMs; (c) PNPs and Pa-PNPs.

of $\mathrm{Pa}-\mathrm{SNPs}$ was used in the binding study. From Figure 5(a), the HA binding ratio was gradually increased as the amount of HA increased. The maximum HA binding ratio of $\mathrm{Pa}$-SNPs around $45 \%$ was achieved. Under the same condition, SNPs without Pa modification only had minimum interaction with $\mathrm{HA}$, suggesting the essential role of $\mathrm{Pa}$ in the binding. Similarly, the maximum $\mathrm{HA}$ binding ratio of $\mathrm{Pa}-$ NEM was about 55\% (Figure 5(b)). Interestingly, the PNPs without surface $\mathrm{Pa}$ had a nonspecific interaction with $\mathrm{HA}$ to induce weak binding between PNPs and HA. Nevertheless, the maximum HA binding ratio of $\mathrm{Pa}-\mathrm{PNPs}$ was around $70 \%$
(Figure 5(c)). The results indicated that although $\mathrm{Pa}$ on the surface of the NPs played an essential role in the binding between HA and NPs, the NPs themselves could also have impact on binding affinity.

We also explored the optimum incubation time for HA binding to the three Pa-NPs. The results indicated the binding was rather fast and maximum binding could be achieved at 15 min (data not shown) for all three Pa-NPs.

The results demonstrated that HA binding to $\mathrm{Pa}-\mathrm{SNPs}$ or $\mathrm{Pa}-\mathrm{NEM}$ gradually increased as the ratio of Pa-Brij 78 in the total surfactant increased from $0 \%$ to $100 \%$, suggesting the 


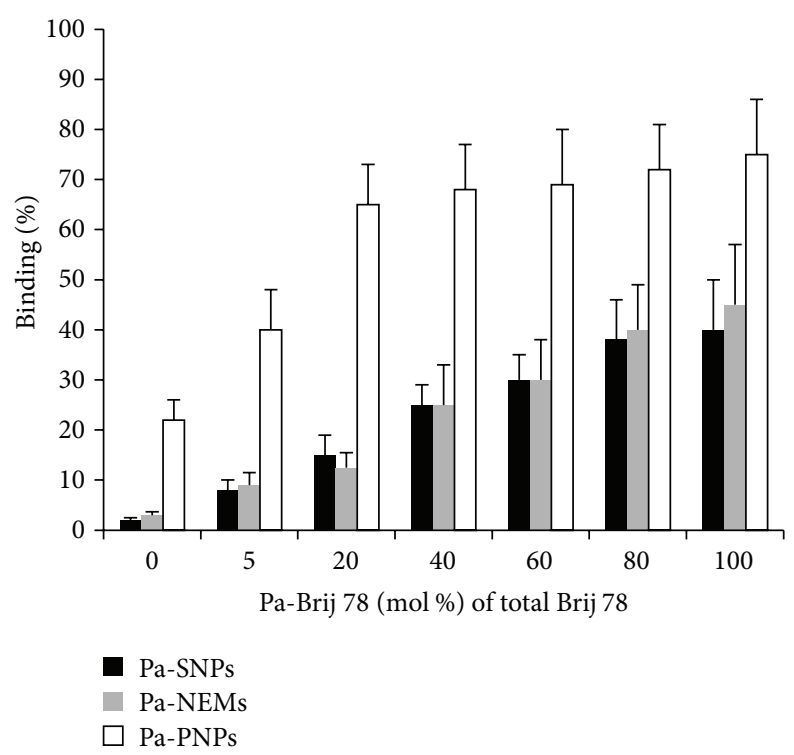

Figure 6: HA binding to Pa-NPs with different molar ratio of Pa-Brij 78 in total surfactant Brij 78 used.

high density of Pa required for the binding. However, the $\mathrm{HA}$ binding to Pa-PNPs achieved maximum as Pa-Brij 78 increased to $20 \%$ of the total surfactant. Further increasing the ratio of Pa-Brij 78 did not change the binding (Figure 6).

The interaction between $\mathrm{Pa}-\mathrm{NPs}$ and $\mathrm{HA}$ to form $\mathrm{HA} / \mathrm{Pa}-$ NPs complex was confirmed by fluorescence microscopy. As shown in Figure 7, HA with fluorescence labeled NPs without surface Pa displayed no or very weak green fluorescence suggesting weak interaction between the NPs and HA. However, each of the Pa-NPs exhibited stronger binding affinity to HA than its parental NPs without Pa. The images in Figure 7 also confirmed that the binding affinity of Pa-PNPs to HA was the highest among all three Pa-NPs.

Lyophilization can keep long-term stability of bioactive and thermosensitive drugs, such as antibiotics and growth factors. After lyophilization and storage in $-80^{\circ} \mathrm{C}$ for a month, the size of recovered $\mathrm{Pa}-\mathrm{SNPs}$ increased to around $280 \mathrm{~nm}$ and the sizes of the other two NPs increased to 200-250 nm (Figure 8(a)). The further optimization of the lyophilization protocol is still ongoing in the lab to obtain satisfying physical stability of NPs. Although the sizes of Pa-NPs increased significantly after the process of freeze-drying, the binding affinities of the three Pa-NPs did not change compared to the fresh prepared Pa-NPs (Figure 8(b)).

\subsection{Characterization of Complex of $\mathrm{HA} / \mathrm{Pa}-\mathrm{NPs}$}

3.4.1. Specific Interaction between $\mathrm{Pa}-\mathrm{NPs}$ and $\mathrm{HA}$. To study the specific interaction between Pa-NPs and HA, the free $\mathrm{Pa}$ was incubated together with the Pa-NPs and HA to test if free $\mathrm{Pa}$ can interfere with the interaction between $\mathrm{Pa}-\mathrm{NPs}$ and $\mathrm{HA}$. The results showed that the $10 \mathrm{mM}$ Pa decreased around $30 \%$ of Pa-NPs bound to HA and $50 \mathrm{mM}$ PA decreased around $65 \%$ of $\mathrm{Pa}-\mathrm{NPs}$ bound to $\mathrm{HA}$ (Figure 9). The results of $\mathrm{Pa}$ concentration dependent competition demonstrated that the binding between Pa-NPs and HA was through the specific interaction between the Pa on the surface of NPs and HA and was independent of the matrix of the NPs. This advantage allows the researchers to choose the appropriate matrix of NPs to encapsulate the drugs of choice. For example, PLGA might be a good candidate for protein based growth factor delivery; the lipid based SNP and NEM could encapsulate hydrophobic drugs. By incorporation of Pa-Brij 78, the three different NPs loaded with different drugs can be incorporated into HA bone graft substitutes. Furthermore, the mixture of the three types of Pa-NPs delivering multiple agents can be loaded simultaneously to HA to form multi-drugs-eluting bone graft materials.

3.4.2. Interference of Free Calcium Ion to the Binding between $\mathrm{Pa}-\mathrm{NPs}$ and HA. The drug loaded HA/Pa-NPs complex was designed as drug-eluting bone graft biomaterials for in vivo use. The affinity mechanism between the Pa-NP and HA is based on the $\mathrm{Pa}$ binding to calcium on the surface of $\mathrm{HA}$, so the free calcium in the body fluid may destroy or decrease the integrity of $\mathrm{HA} / \mathrm{Pa}-\mathrm{NPs}$. Therefore, we first used the blood concentration of calcium to study its effects on the stability of prepared HA/Pa-NPs. The results showed that the $2.5 \mathrm{mM}$ calcium chloride (equal to normal Ca concentration in serum) did not break down the HA/Pa-NPs, suggesting the complex can tolerate the free calcium in the body fluid. However, if the concentration of calcium was increased to $5.0 \mathrm{mM}$, the majority of the NPs would have been released from the complex (Figure 10(a)).

Because HA crystal is a mimic of natural bone, the study of in vitro $\mathrm{Pa}-\mathrm{NPs}$ targeting $\mathrm{HA}$ with the existence of $\mathrm{Ca}^{2+}$ can preliminarily test if $\mathrm{Pa}-\mathrm{NPs}$ are suitable for targeted delivery of drugs to bones. The results demonstrated that $\mathrm{Pa}-\mathrm{SNPs}$ and $\mathrm{Pa}-\mathrm{NEM}$ lost their affinity to $\mathrm{HA}$ with the $\mathrm{Ca}^{2+}$ concentration in serum $(2.5 \mathrm{mM})$, so these Pa-NPs are not suitable for 

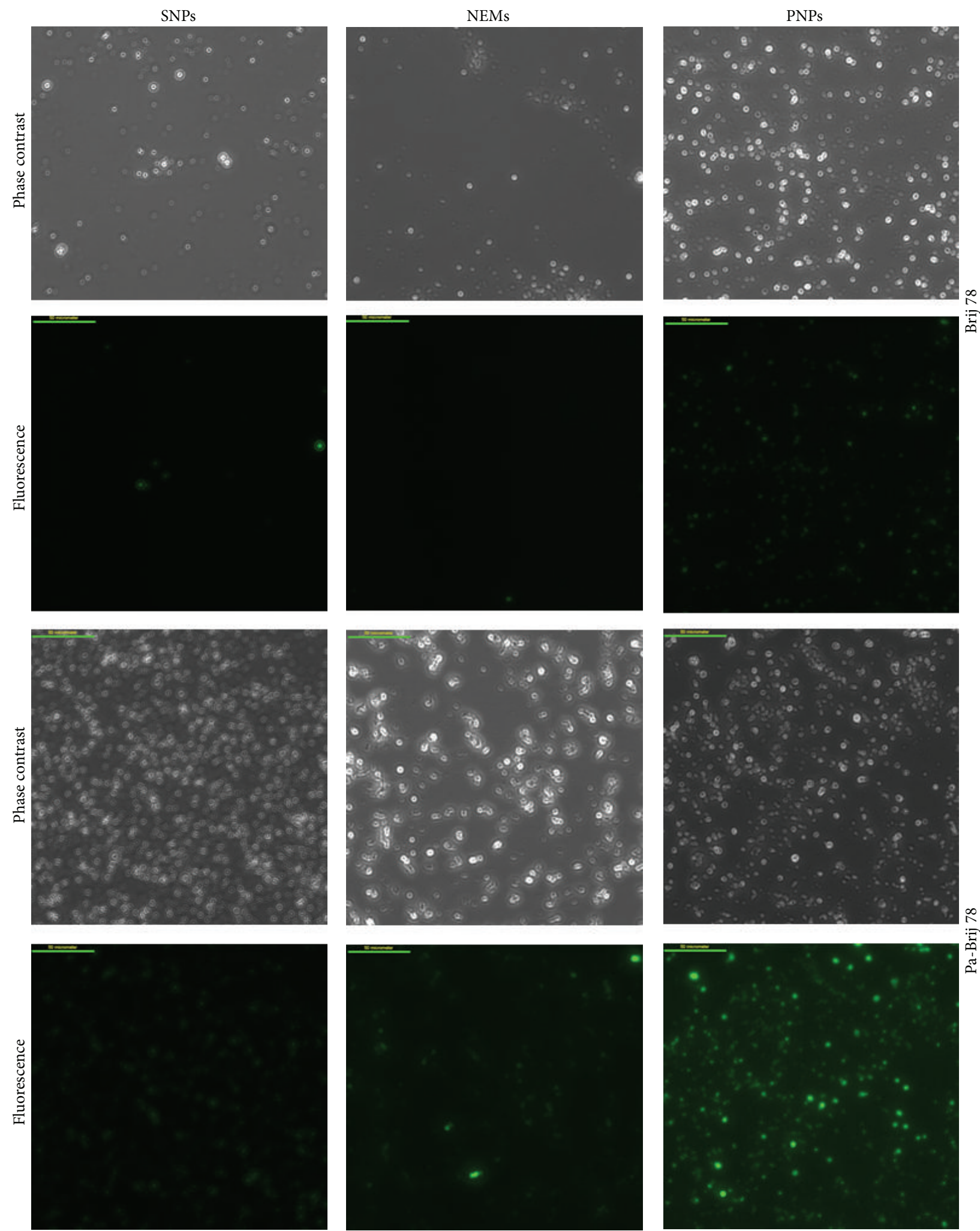

(a)

(b)

(c)

FIGURE 7: Fluorescence images of HA/Pa-NPS. The NPs were labeled with coumarin described in Section 2. (a) SNPs and Pa-SNPs; (b) NEMs and Pa-NEMs; (c) PNPs and Pa-PNPs. The phase contrast and fluorescence images were captured by Olympus IX71 fluorescence microscope. 


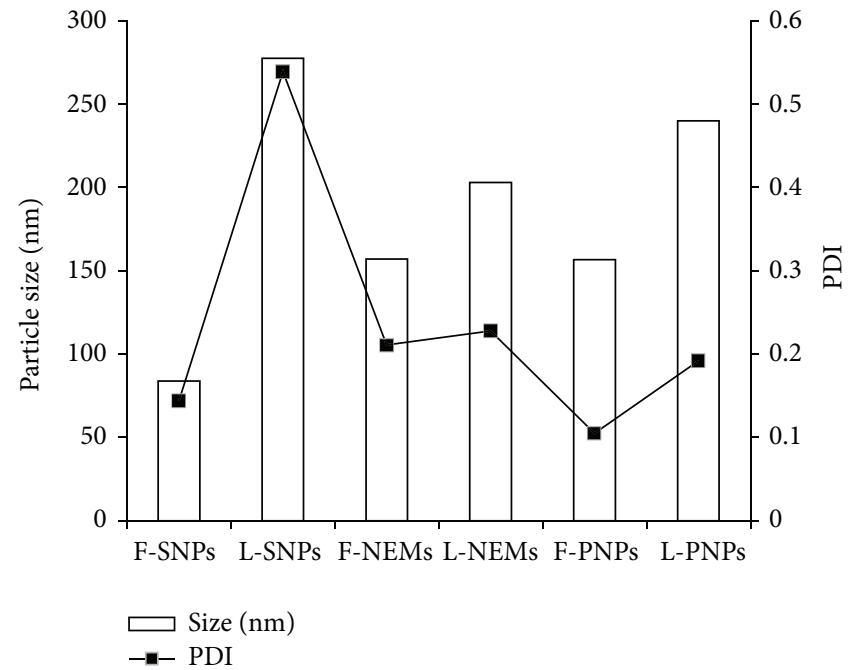

(a)

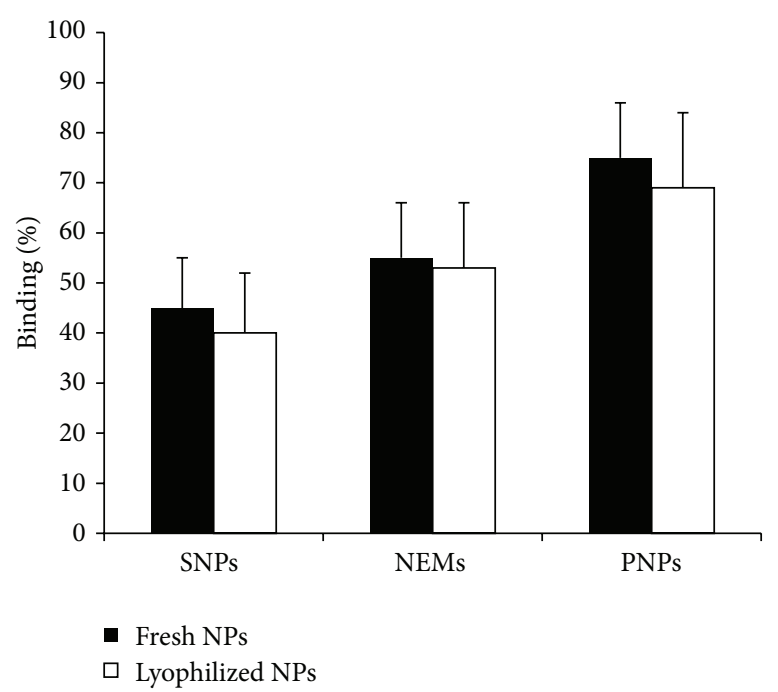

(b)

FIGURE 8: The physical property and binding affinity of the fresh (F) prepared and lyophilized (L) Pa-NPs. (a) Particle size and PDI of Pa-NPs. (b) Binding affinities of Pa-NPs to HA.

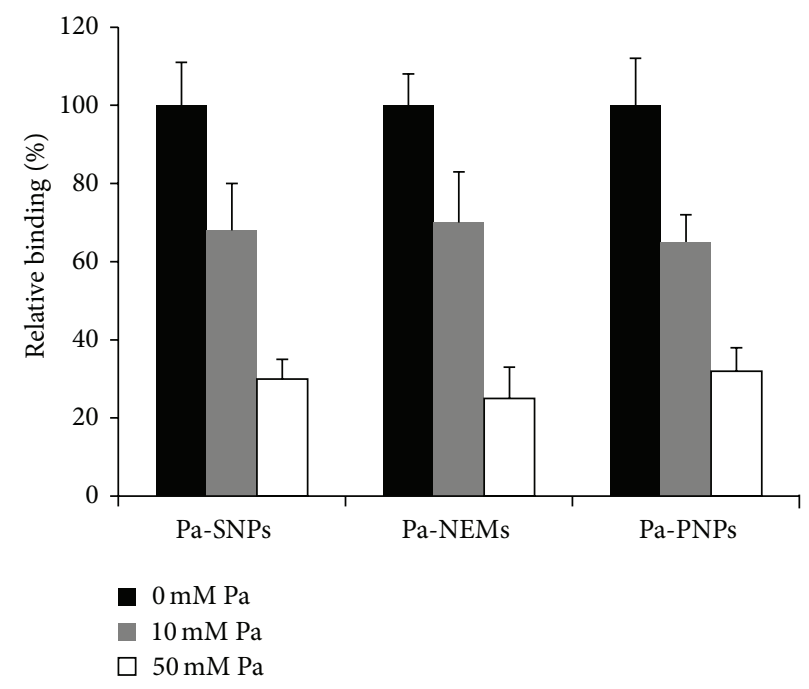

Figure 9: The HA binding to Pa-NPs with existance of free Pa.

bone targeting through i.v. administration. However, the PaPNPs still interacted with HA with the existence of $2.5 \mathrm{mM}$ calcium chloride, suggesting that Pa-PNPs have the potential to deliver therapeutic agents for treatment of bone diseases and tumor bone metastasis (Figure 10(b)).

\section{Conclusions}

In conclusion, a Pa-modified Brij 78 was synthesized and the new compound Pa-Brij 78 was used as both surfactants and a target ligand for fabrication of a series of drug loaded Pa-NPs which could interact with HA. The complex of HA/Pa-NPs has potential to construct drug or growth factor eluting HAbased bone graft substitute.

\section{Conflict of Interests}

The authors declare that there is no conflict of interests regarding the publication of this paper.

\section{Acknowledgment}

This work was supported by Construction Program for Innovative Research Team of University in Sichuan Province (14TD0050). 


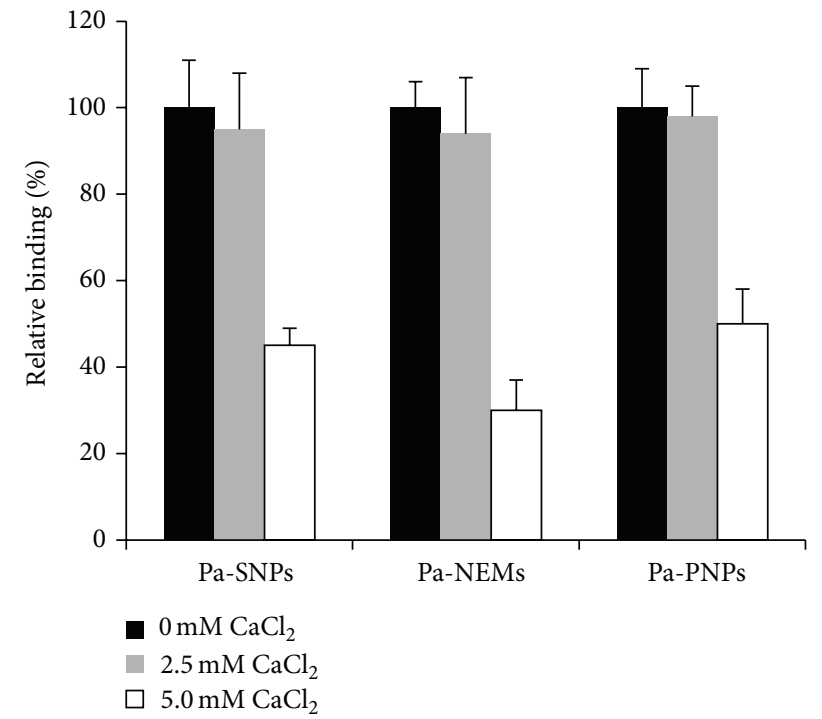

(a)

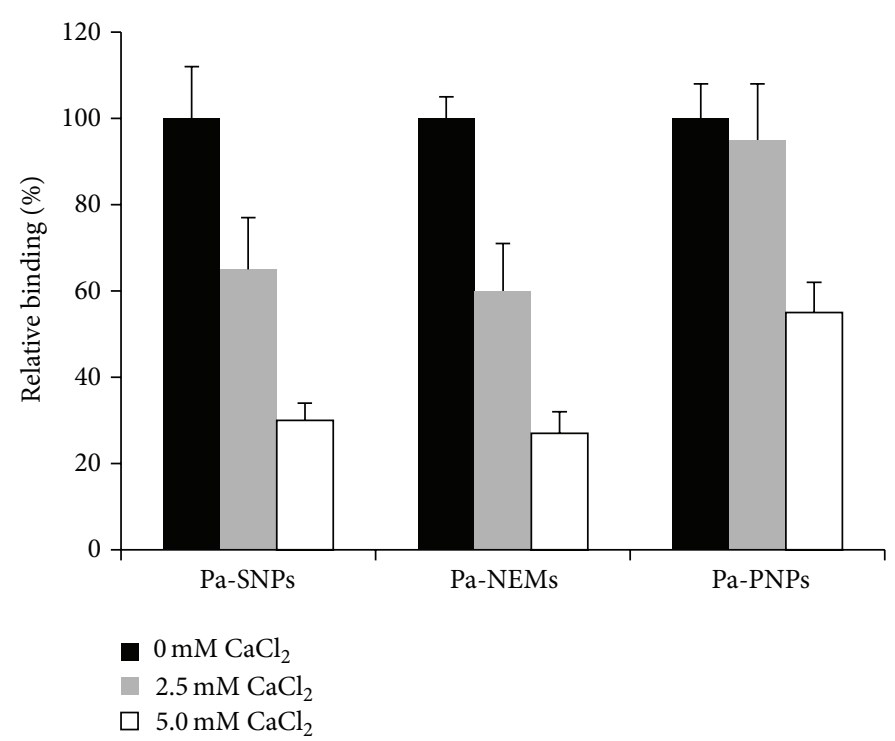

(b)

Figure 10: The effects of free calcium ion on the HA binding to Pa-NPs. (a) The effects of free calcium on the integrity of prepared complex of HA/Pa-NPs. (b) The effects of free calcium on the in vitro binding of Pa-NP to HA.

\section{References}

[1] L. L. Hench, "Bioceramics-from concept to clinic," Journal of the American Ceramic Society, vol. 74, pp. 1487-1510, 1991.

[2] S. Itoh, S. Nakamura, M. Nakamura, K. Shinomiya, and K. Yamashita, "Enhanced bone ingrowth into hydroxyapatite with interconnected pores by Electrical Polarization," Biomaterials, vol. 27, no. 32, pp. 5572-5579, 2006.

[3] P. Wu and D. W. Grainger, "Drug/device combinations for local drug therapies and infection prophylaxis," Biomaterials, vol. 27, no. 11, pp. 2450-2467, 2006.

[4] M. Brown, Y. Zou, R. Peyyala, T. Dziubla, and D. Puleo, “Temporal separation in the release of bioactive molecules from a moldable calcium sulfate bone graft substitute," Current Drug Delivery, vol. 11, no. 5, pp. 605-612, 2014.

[5] S. P. Becerra, L. A. Perez-Mediavilla, J. E. Weldon et al., "Pigment epithelium-derived factor binds to hyaluronan: mapping of a hyaluronan binding site," The Journal of Biological Chemistry, vol. 283, no. 48, pp. 33310-33320, 2008.

[6] F.-H. Lin, G.-C. Dong, K.-S. Chen, G. J. Jiang, C.-W. Huang, and J.-S. Sun, "Immobilization of Chinese herbal medicine onto the surface-modified calcium hydrogenphosphate," Biomaterials, vol. 24, no. 13, pp. 2413-2422, 2003.

[7] S. E. Bae, J. Choi, Y. K. Joung, K. Park, and D. K. Han, "Controlled release of bone morphogenetic protein (BMP)-2 from nanocomplex incorporated on hydroxyapatite-formed titanium surface," Journal of Controlled Release, vol. 160, no. 3, pp. 676684, 2012.

[8] J. Balzeau, M. Pinier, R. Berges, P. Saulnier, J.-P. Benoit, and J. Eyer, "The effect of functionalizing lipid nanocapsules with NFL-TBS.40-63 peptide on their uptake by glioblastoma cells," Biomaterials, vol. 34, no. 13, pp. 3381-3389, 2013.

[9] M. P. Siqueira-Moura, F. L. Primo, E. M. Espreafico, and A. C. Tedesco, "Development, characterization, and photocytotoxicity assessment on human melanoma of chloroaluminum phthalocyanine nanocapsules," Materials Science and Engineering C: Materials for Biological Applications, vol. 33, no. 3, pp. 1744-1752, 2013.

[10] A. Yadav, V. Lomash, M. Samim, and S. J. S. Flora, "Curcumin encapsulated in chitosan nanoparticles: a novel strategy for the treatment of arsenic toxicity," Chemico-Biological Interactions, vol. 199, no. 1, pp. 49-61, 2012.

[11] W. Yan and L. Huang, "Recent advances in liposome-based nanoparticles for antigen delivery," Polymer Reviews, vol. 47, no. 3, pp. 329-344, 2007.

[12] K. R. Chaudhari, A. Kumar, V. K. M. Khandelwal et al., "Bone metastasis targeting: a novel approach to reach bone using Zoledronate anchored PLGA nanoparticle as carrier system loaded with Docetaxel," Journal of Controlled Release, vol. 158, no. 3, pp. 470-478, 2012.

[13] L. de Miguel, M. Noiray, G. Surpateanu, B. I. Iorga, and G. Ponchel, "Poly(gamma-benzyl-L-glutamate)-PEG-alendronate multivalent nanoparticles for bone targeting," International Journal of Pharmaceutics, vol. 460, no. 1-2, pp. 73-82, 2014.

[14] V. Hengst, C. Oussoren, T. Kissel, and G. Storm, "Bone targeting potential of bisphosphonate-targeted liposomes: preparation, characterization and hydroxyapatite binding in vitro," International Journal of Pharmaceutics, vol. 331, no. 2, pp. 224-227, 2007.

[15] S. I. Thamake, S. L. Raut, Z. Gryczynski, A. P. Ranjan, and J. K. Vishwanatha, "Alendronate coated poly-lactic-co-glycolic acid (PLGA) nanoparticles for active targeting of metastatic breast cancer," Biomaterials, vol. 33, no. 29, pp. 7164-7173, 2012.

[16] Y. Dong and S.-S. Feng, "Poly(D,L-lactide-co-glycolide) (PLGA) nanoparticles prepared by high pressure homogenization for paclitaxel chemotherapy," International Journal of Pharmaceutics, vol. 342, no. 1-2, pp. 208-214, 2007.

[17] M. Ao, G. Xu, Y. Zhu, and Y. Bai, "Synthesis and properties of ionic liquid-type Gemini imidazolium surfactants," Journal of Colloid and Interface Science, vol. 326, no. 2, pp. 490-495, 2008.

[18] L. Feng, H. L. Wu, P. Ma, R. J. Mumper, and S. R. Benhabbour, "Development and optimization of oil-filled lipid nanoparticles 
containing docetaxel conjugates designed to control the drug release rate in vitro and in vivo," International Journal of Nanomedicine, vol. 6, pp. 2545-2556, 2011.

[19] A. Jain, W. Yan, K. R. Miller, R. O’Carra, J. G. Woodward, and R. J. Mumper, "Tresyl-based conjugation of protein antigen to lipid nanoparticles increases antigen immunogenicity," International Journal of Pharmaceutics, vol. 401, no. 1-2, pp. 87-92, 2010. 

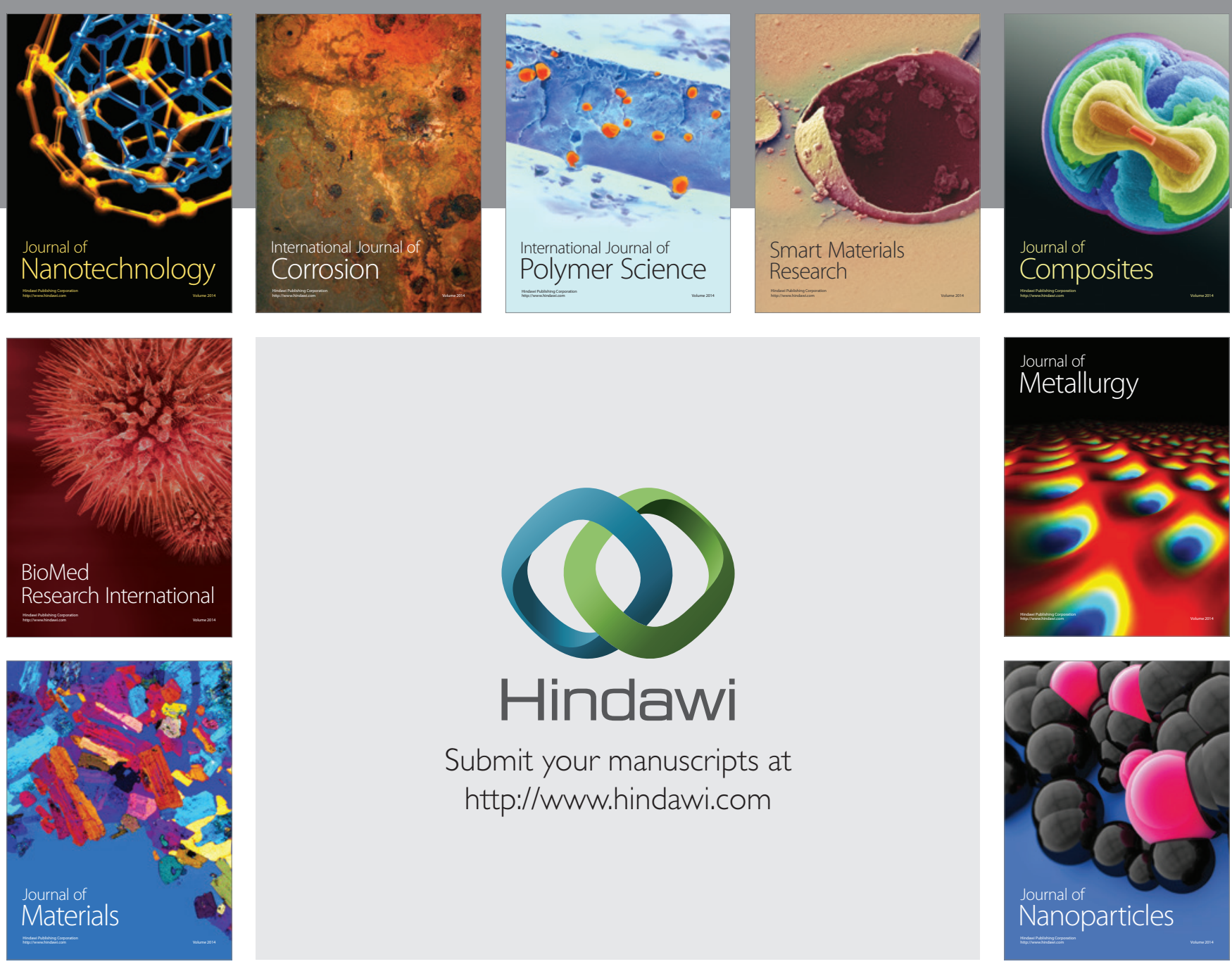

Submit your manuscripts at http://www.hindawi.com
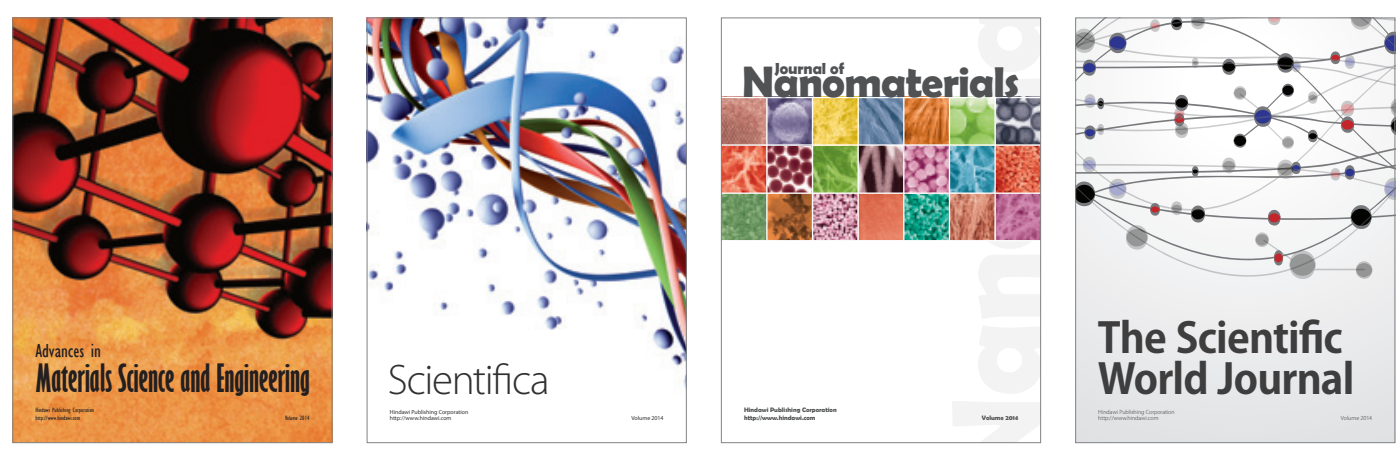

\section{The Scientific World Journal}
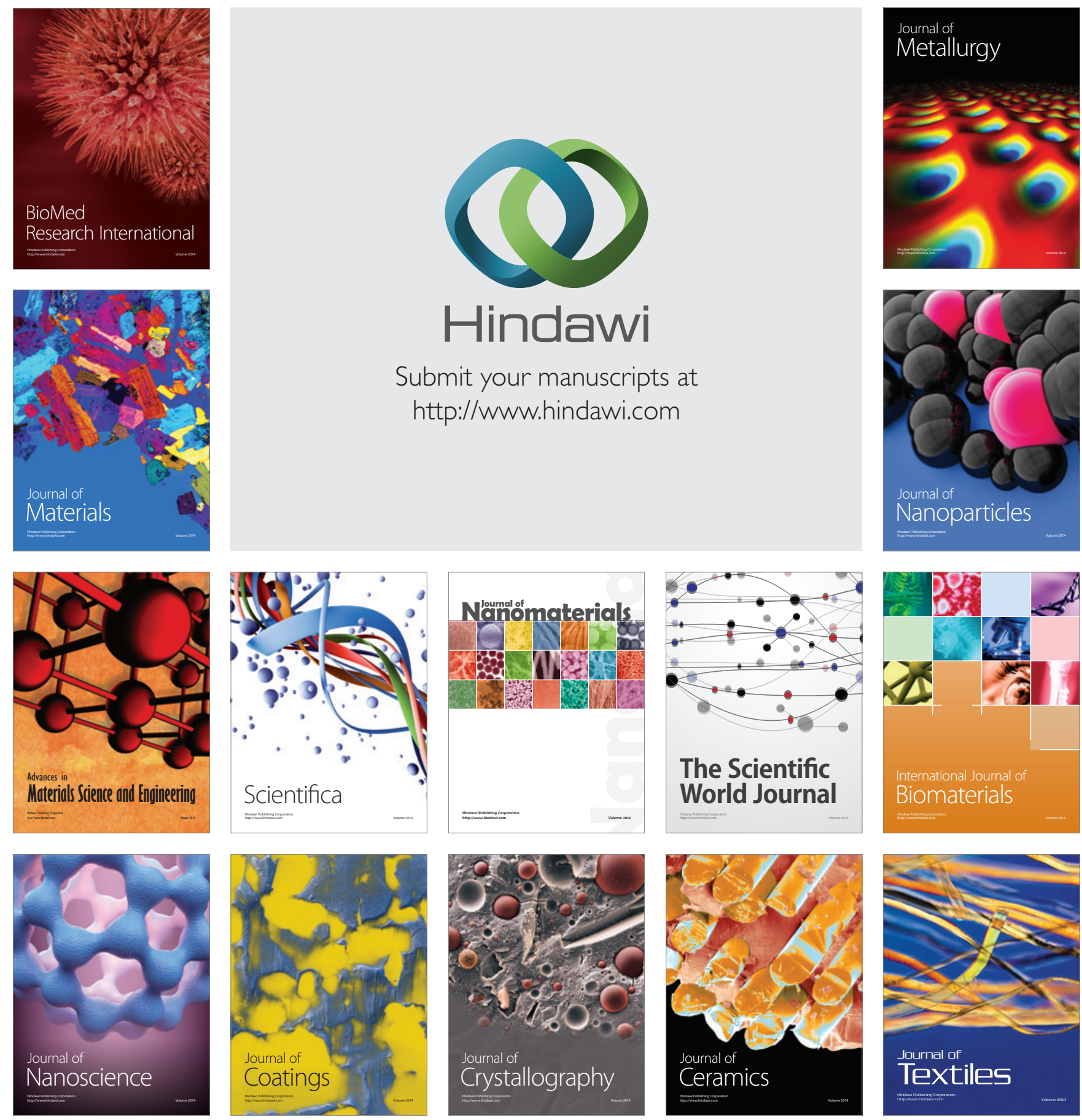